\title{
Effects of desferrioxamine in patients with iron-loading with a simple method for estimating urinary iron
}

\author{
M. S. LOSOWSKY \\ From the Department of Medicine, The General Infirmary, Leeds
}

SYNOPSIS Desferrioxamine produced marked increases in urinary iron excretion in three patients with very gross iron loading. The magnitude of the response to desferrioxamine was not closely related to the amount of iron seen in liver biopsy sections. In two patients with less severe iron loading, desferrioxamine produced no significant increase in urinary iron content. In the patients who responded, the effect of desferrioxamine did not diminish with repeated doses even over periods of up to a year.

The effectiveness of desferrioxamine was not increased by intravenous administration, water diuresis, mersalyl, or ammonium chloride.

A simple method for estimation of urinary iron is described, necessitating a minimum of glassware, and sufficiently accurate for clinical purposes.

In idiopathic haemochromatosis, deposition of iron in the tissues is associated with cirrhosis of the liver and with damage in other organs. Excessive iron deposition may also occur secondarily to prolonged anaemia of various types (Zeltmacher and Bevans, 1945; Goldish and Aufderheide, 1953; Gelpi and Ende, 1958), and in some such patients tissue damage, including cirrhosis of the liver, may follow. It is not certain that iron itself is the cause of liver damage (MacDonald, 1964) but, in view of this possibility, consideration must be given to methods for the removal of iron from the body. In the presence of anaemia it is unlikely that frequent venesection will be possible and thus some other method is necessary.

Desferrioxamine is a chelating agent with a remarkable affinity and specificity for iron (Tripod, 1964) and has been shown to cause considerable increases in its excretion in patients with iron loading (Moeschlin and Schnider, 1963; Walsh, Mass, Smith, and Lange, 1964a). The amount of iron lost, however, is rarely as great as can be achieved by venesection and we have investigated some possible ways of improving the effectiveness of desferrioxamine. In addition, since therapy would need to be prolonged we have assessed the response to repeated courses and to prolonged use.

Received for publication 27 October 1965.
Furthermore, the usual methods for estimation of urinary iron can be time-consuming, and necessitate the use of iron-free glassware, and a simple and easy method of estimating iron excretion within the limits of accuracy which are of clinical importance is described.

\section{PATIENTS STUDIED}

Five patients were studied. All showed a gross excess of iron on appropriate staining of liver biopsy sections, and all had a considerable amount of 'free' iron on bone marrow smears. In addition, the serum iron and percentage saturation of iron-binding capacity were above normal in all.

In three of the patients the excess iron in the liver was extremely large and all these had cirrhosis, but none was jaundiced. One of these (patient 1) had acquired sideroblastic anaemia, another (patient 2) had an unusual defect of iron metabolism with hypochromic anaemia and virtually no iron in the erythroblasts, and the third (patient 3) had idiopathic haemochromatosis. This last patient undoubtedly had the greatest amount of iron of all in his liver biopsy. In the remaining two patients the iron deposition in the liver was not as extensive, but nevertheless was grossly abnormal, and there was no cirrhosis. One of these patients had hereditary sideroblastic anaemia (Losowsky and Hall, 1965) 
and the other had chronic haemolytic anaemia of unknown cause.

\section{METHODS}

Twenty-four hour samples of urine were collected directly into acid-washed jars, and aliquots taken after mixing. All urine collections were made in hospital excepting the later ones of the patient treated for many months, and these out-patient collections appeared to be reliable.

In the tests involving hydration and dehydration, patients were asked to drink as much as possible or restricted to $500 \mathrm{ml}$. of fluid per 24 hours respectively. This was started in each case 12 hours before urine collection was begun and continued for 48 hours. The order of these procedures was alternated, and there was at least $\mathbf{4 8}$ hours of free fluid intake for the patient between one procedure and the other. The samples for each 48hour period were pooled, and the iron excretion expressed as the mean value per day.

Mersalyl was given in a dosage of $2 \mathrm{ml}$. intramuscularly at the start of the daily urine collection.

Ammonium chloride in a dosage of $2 \mathrm{~g}$. three times per day was given in liquid form.

Desferrioxamine methane sulphonate was used. Intramuscular injections were given in $5 \mathrm{ml}$. of water at the start of the daily urine collection and, where indicated, also 12 hours later. Intravenous doses were given by infusion in transfused blood, starting at the beginning of the daily urine collection, each $500 \mathrm{mg}$. being spread over six to eight hours.

Urinary iron concentrations were measured by the method of Peters, Giovanniello, Apt, and Ross (1956) with a correction for other pigments present being made by adjusting the blank reading for each sample separately as described by Henry, Sobel, and Chiamori (1958). Readings were compared with standard solutions of both iron and ferrioxamine.

The simple method for estimation of urinary iron is performed as follows. Spectrophotometer cuvettes $(1 \mathrm{~cm}$. light path) kept in $50 \%$ nitric acid are rinsed well with ordinary distilled water and dried. Using an acid-washed pipette, $3 \mathrm{ml}$. of urine is placed in the cuvette. From a dropper bottle, 6 drops of $80 \%$ thioglycollic acid is added, a piece of paraffin film is held over the top, the cuvette inverted two or three times, and read in the spectrophotometer at $520 \mathrm{~m} \mu$. From another dropper bottle 6 drops of $1 \%(\mathrm{w} / \mathrm{v}) \alpha \alpha^{1}$ dipyridyl in $0.5 \mathrm{~N}$ sulphuric acid is added, the cuvette again inverted two or three times and read again in the spectrophotometer at $520 \mathrm{~m} \mu$ after 10 minutes. The difference in the two readings is compared with those obtained from one or more standard solutions of iron or ferrioxamine. If the iron concentration is above about $1,200 \mu \mathrm{g} . \%$ or if there is considerable other pigment in the urine, it may be necessary to dilute the sample before testing.

Results are not markedly altered if the $p \mathrm{H}$ of the sample varies from 2.5 to $10 \cdot 0$. The solutions of thioglycollic acid and $\alpha \alpha^{1}$ dipyridyl can be stored at room temperature, the only deterioration being that after storage of the dipyridyl reagent for some weeks the colour may take
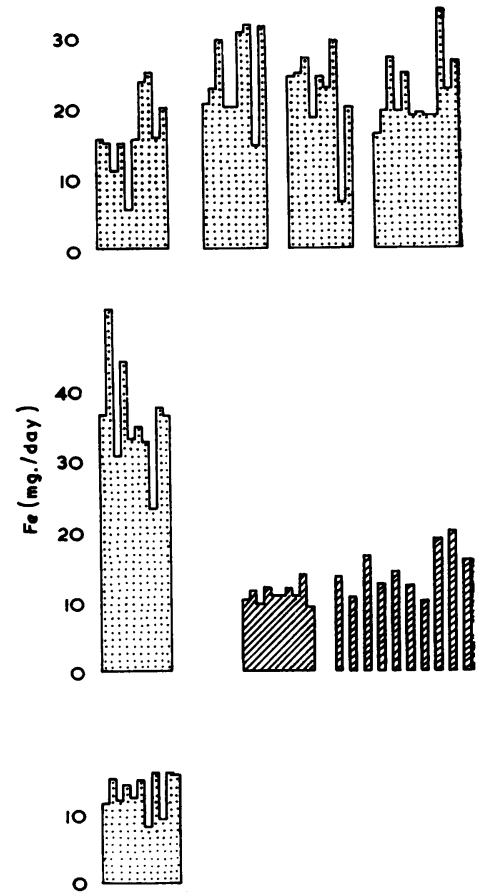

FIG. 1. Urinary iron excretion after desferrioxamine. Top diagrams represent daily excretions of iron in case 1 , with successive courses of desferrioxamine, $500 \mathrm{mg}$. twice daily $\overline{\mathrm{D}}$ intramuscularly. The four courses were spread over a total 0 duration of 15 weeks. There was no diminution in effective- $\stackrel{\mathbb{Q}}{\varrho}$ ness during each course, or in successive courses.

Middle diagrams represent case 2. The first course was at a dosage of $500 \mathrm{mg}$. twice daily. The second course shows the smaller response to $500 \mathrm{mg}$. daily. Treatment was continued at this dose and single columns represent 24-hour excretions at intervals of one month, showing no diminution of effectiveness.

The bottom diagram represents the response to $500 \mathrm{mg}$. twice daily in case 3, the patient who had the greatest degree of iron loading of the three but showed the smallest 윽 iron excretion at this dose.

up to 45 minutes to develop but the final intensity is unchanged.

\section{RESULTS}

EFFECT OF DESFERRIOXAMINE ON IRON EXCRETION Control observations of urinary iron excretion were performed on numerous occasions on all patients, and were always less than $2 \mathrm{mg}$. per day and usually below $1 \mathrm{mg}$. per day.

Figure 1 shows urinary iron excretion after desferrioxamine in the three patients with the $\frac{\vec{\mathbb{}}}{\mathrm{O}}$ greatest iron loading in the liver.

Figure 1 shows the effects of successive courses of $\mathbb{D}$ desferrioxamine in cases 1,2 , and 3 . It can be seen?

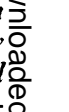

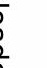


that in case 1 there is no diminution in iron excretion during each course nor is there any decrease in effectiveness from one course of treatment to the next. In case 2 the lower dose was obviously less effective but in neither case did the effectiveness diminish during the course. It can be seen also that iron excretion did not diminish over the space of almost a year. Again in case 3 the effectiveness did not diminish during the course. The mean daily iron excretion is considerably lower than in the first two patients, even though the degree of iron loading in the liver of this patient was clearly the most severe of all.

In all cases, iron excretion was down almost to control levels on the day after desferrioxamine was stopped.

In the remaining two patients increase in iron excretion on $500 \mathrm{mg}$. desferrioxamine twice daily was less than $1 \mathrm{mg}$. per day despite well-marked iron loading on liver biopsy.

Toxic effects of desferrioxamine were not encountered, with the possible exception of lenticular opacities in both eyes in case 2 while on long-term treatment. These improved when desferrioxamine was stopped. In cases 1 and 2, marked improvement in skin pigmentation was noted in the early weeks of treatment.

ATTEMPTS TO INCREASE THE EFFECTIVENESS OF DESFERRIOXAMINE All these studies were carried out while desferrioxamine had been given intramuscularly in the same dosage for at least three days preceding the start of the test.

Intravenous infusion of desferrioxamine in two patients was rather less effective than intramuscular injection (Fig. 2).

Iron excretion during a water diuresis was greater than during fluid restriction on each of four occasions in three patients (Fig. 3). The increase in iron excretion during water diuresis, however, was by no means proportional to the urine volume, and in each case the mean excretion during water diuresis was no greater than that obtained by allowing a free fluid intake in the same patient. Although restriction of fluid intake appeared to reduce the iron excretion, there was no compensatory increase in excretion in ensuing 24-hour samples.

Ammonium chloride given for two successive days to two patients (Fig. 4) produced no significant differences either in urine volume or in iron excretion when compared with the immediately preceding and succeeding days. The single columns on the right of Fig. 4 show the effect, in case 2, of ammonium chloride given for three days each week. Five weekly 24-hour samples were taken on the third day of administration of ammonium chloride, and com-
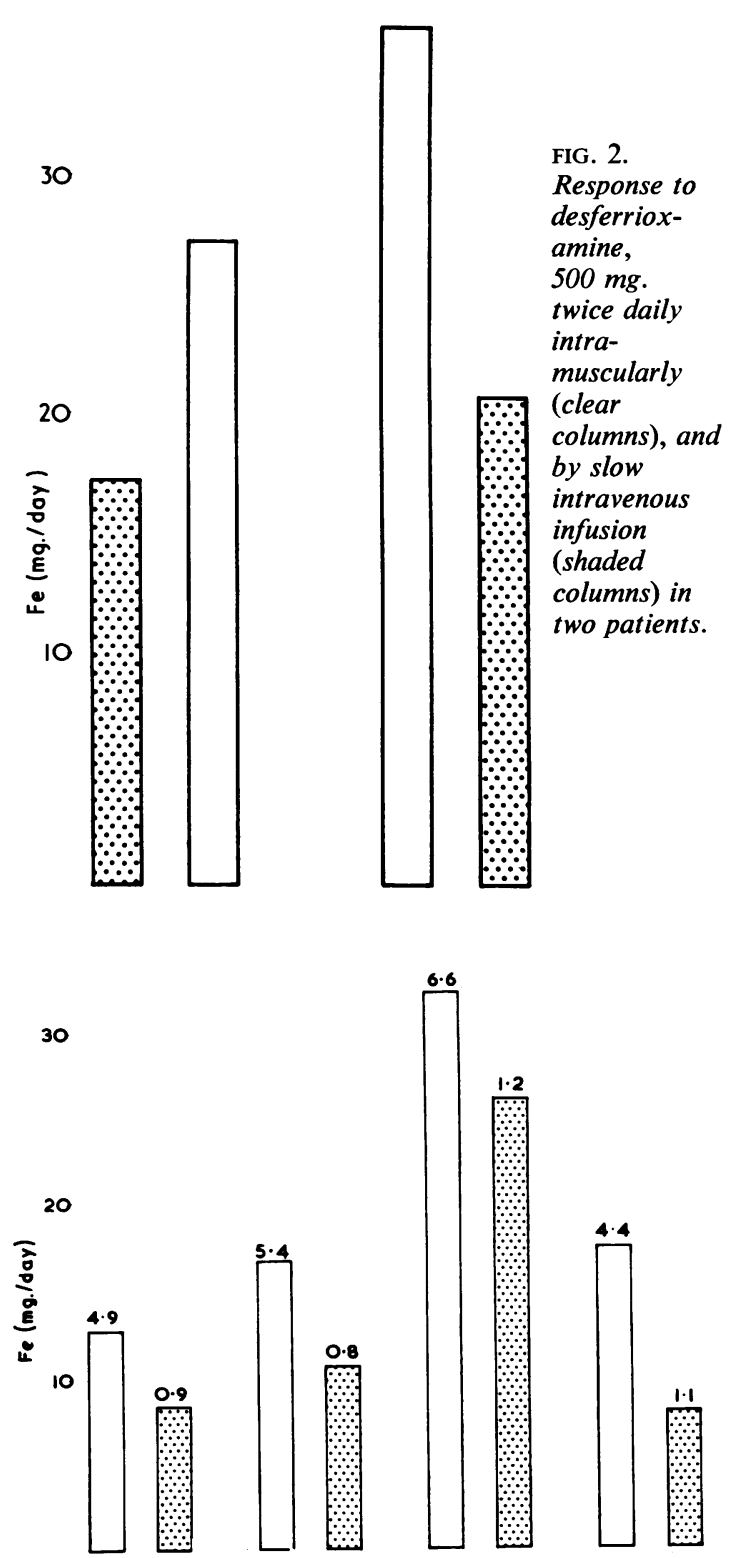

FIG. 3. Responses to desferrioxamine during a water diuresis (clear columns) and during fluid restriction (shaded columns). Each column represents the mean daily iron excretion over a 48-hour period, the mean daily urine volume (in litres) being written above. The order of the procedures was alternated, at least two days of free fluid intake being allowed between them. The middle two sets of results were both on case 2, at doses of $500 \mathrm{mg}$. daily and $500 \mathrm{mg}$. twice daily, the other results being obtained with $500 \mathrm{mg}$. daily in cases 1 and 3 respectively.

There was some reduction in iron excretion with fluid restriction. 


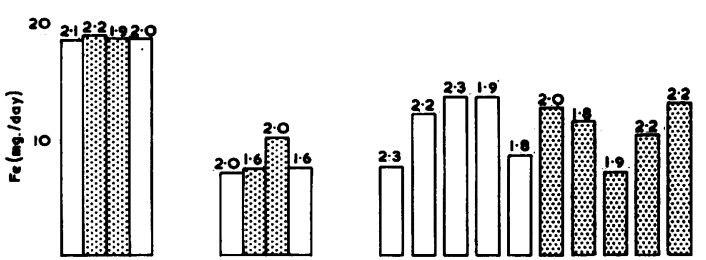

FIG. 4. Effects of ammonium chloride $2 \mathrm{~g}$. three times daily (hatched columns) on iron excretion during desferrioxamine $500 \mathrm{mg}$. daily in three patients. Urine volumes (litres) are written above each column. The first two diagrams show no difference in urine volume or iron excretion as compared with preceding or succeeding days. The single columns represent weekly 24-hour samples during 10 weeks. For the last five samples ammonium chloride was given for three days each week and the sample taken on the third day. There was no effect on urine volume or iron excretion.

pared with the five preceding weekly 24-hour samples without ammonium chloride. It can be seen that there is no significant difference in urine volume or iron excretion.

In Fig. 5 are shown the results of the administration of mersalyl on five occasions to three patients, when compared with the days immediately preceding and succeeding. On each occasion the 24-hour urinary output was greater after the mersalyl, but there was no significant increase in iron excretion.

SIMPLE METHOD FOR ESTIMATION OF URINARY IRON Figure 6 shows the correlation between the results of the two methods of measurement of iron con-
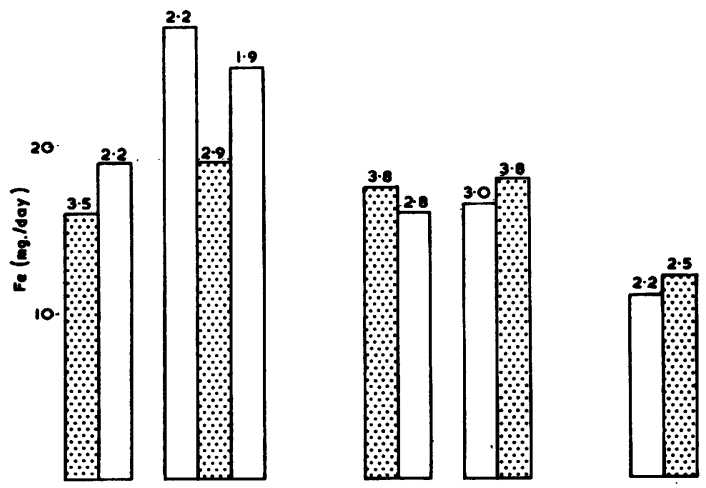

FIG. 5. Effect of mersalyl (shaded colums) during administration of desferrioxamine $500 \mathrm{mg}$. twice daily on five occasions in three patients. Urine volume increased but iron excretion was unchanged as compared with preceding or succeeding days. centration on 135 unselected samples of urine fro these five patients. The values obtained by the two methods are closely comparable in all except two samples in which the simple method overestimated the iron content. The results, however, are sufficienty comparable to suggest that the simple method adequate for clinical purposes.

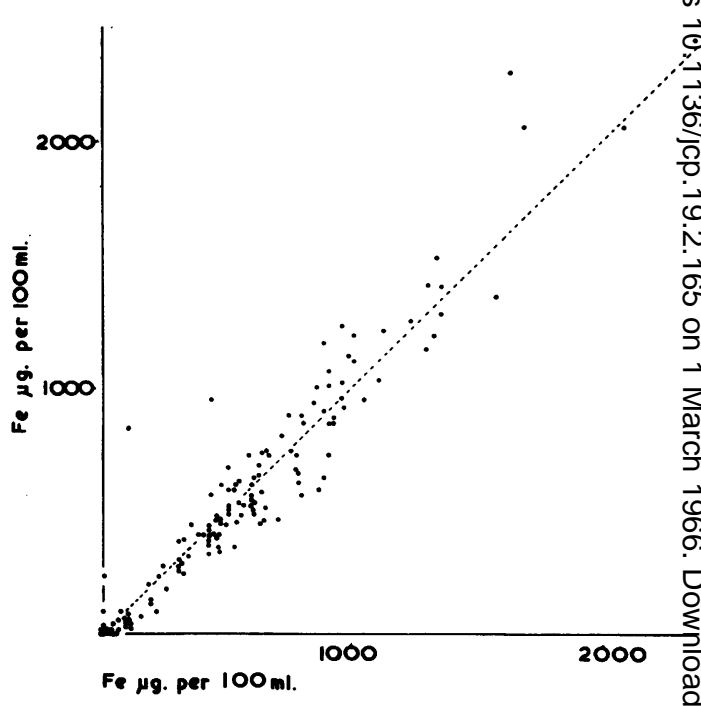

FIG. 6. Comparison of urinary iron concentration $\underset{\mathbb{Q}}{\mathbb{Q}} \mathrm{s}$ measured by the method of Peters, Giovanniello, Apt, ated Ross (1956) on the horizontal axis, and the simple methed described here, on the vertical axis. The simple method gives results of sufficient accuracy for clinical purposes

\section{DISCUSSION}

Desferrioxamine has previously been shown to be effective in removing iron from the body in patiegts with iron-loading occurring in idiopathic haemsochromatosis or refractory anaemia (Moeschlin and Schnider, 1963; Wöhler, 1963; Hwang and Brow 1964; Walsh et al., 1964a), and this has been borñ out in the present study.

In some patients with anaemia venesection maynbe possible (Finch and Barnett, 1957; Crosby and Sheehy, 1960; Byrd and Cooper, 1961) but this obviously cannot be undertaken in the severely anaemic patient, and here desferrioxamine represents the only means of removing iron. The amountof iron removed, however, may average only 10 to $20 \mathrm{mg}$. per day, compared with the $35 \mathrm{mg}$. a day removed by a weekly venesection of 1 pint of blogd, if there is no anaemia. Furthermore, the discomf ofrt and inconvenience of a daily or twice daily intramuscular injection and the possible variability of 
effectiveness of desferrioxamine from patient to patient must be taken into account.

It has been suggested that the iron excretion in response to a chelating agent may be an indication of the degree of iron loading in the tissues (Walsh, Perkins, Blackburn, Sanford, and Cantrill, 1963; Fielding, 1965). In our patients, the average daily iron excretion when on a regular dose of desferrioxamine did not correlate well with the degree of iron loading, in that our most severely affected patient showed a rather low excretion, and two of our iron-loaded subjects, albeit the least severely affected, showed no significant iron excretion. It may be that a significant iron excretion with desferrioxamine can be taken as an indication of very gross iron loading, or perhaps the fact that each of these three patients also had cirrhosis of the liver may have been relevant.

Although haematological improvement has been recorded after desferrioxamine (Wöhler, 1964b; Moeschlin and Schnider, 1963), we have not been able to confirm this in any of our anaemic patients, despite careful and repeated evaluation.

The specificity of desferrioxamine for iron is of great importance since, except in acute iron poisoning, prolonged use will presumably be necessary and a less specific chelating agent might entail a risk of producing deficiencies of other elements (Hwang and Brown, 1964). From information so far available (Hwang and Brown, 1964; Moeschlin and Schnider, 1963) there seems no loss of effectiveness of desferrioxamine even over periods of many months, and we have confirmed this.

We have been unable to produce any significant increase in effectiveness of desferrioxamine by intravenous infusion (Hwang and Brown, 1964; Smith, 1964), water diuresis, a mercurial diuretic, or ammonium chloride. In this last point we differ from Walsh et al. (1964a) who observed an increase in iron excretion with ammonium chloride, and the reason for this difference is not clear.
The simple method of estimating urinary iron includes a colour reaction for iron rather than measurement of decolorization produced by sequestrine as described by Fielding and Brunström (1964) and is presumably relatively specific. The procedure has deliberately been simplified for routine use, omitting centrifugation or buffering, and using a minimum of glassware. Accuracy still seems to be sufficient for clinical purposes. Results compare well with the method of Peters et al. (1956) and although this may be open to some objections in that preliminary digestion of the urine was not performed (Hwang and Brown, 1964), we feel from experiments of recovery of iron and ferrioxamine in vitro that the results are reliable. Thus, for clinical purposes there is a simple method of estimation of the effectiveness of desferrioxamine which would be of value in assessment of long-term treatment.

I thank Ciba Ltd. for initial supplies of desferrioxamine, and Miss C. Shaw for expert technical assistance.

\section{REFERENCES}

Byrd, R. B., and Cooper, T. (1961). Ann. intern. Med., 55, 103.

Crosby, W. H., and Sheehy, T. W. (1960). Brit. J. Haemat., 6, 56. Fielding, J. (1965). J. clin. Path., 18, 88.

and Brunström, G. M. (1964). Ibid., 17, 395.

Finch, S. C., and Barnett, R. N. (1957). New Engl. J. Med., 256, 884.

Gelpi, A. P., and Ende, N. (1958). Amer. J. Med., 25, 303.

Goldish, R. J., and Aufderheide, A. C. (1953). Blood, 8, 837.

Henry, R. J., Sobel, C., and Chiamori, N. (1958). Clin. chim. Acta, 3 , 523.

Hwang, Y.-F., and Brown, E. B. (1964). Arch. intern. Med., 114, 741

Losowsky, M. S., and Hall, R. (1965). Brit. J. Haemat., 11, 70.

MacDonald, R. A. (1964). Hemochromatosis and Hemosiderosis. Thomas, Springfield, Illinois.

Moeschlin, S., and Schnider, U. (1963). New Engl. J. Med., 269, 57.

Peters, T., Giovanniello, T. J., Apt, L., and Ross, J. F. (1956). J. Lab. clin. Med., 48, 280.

Smith, R. S. (1964). Ann. N.Y. Acad. Sci., 119, 776.

Tripod, J. (1964). In Iron Metabolism, edited by F. Gross, pp. 503-524. Springer, Berlin.

Walsh, J. R., Mass, R. E., Smith, F. W., and Lange, V. (1964a) Arch. intern. Med., 113, 435.

Walsh, R. J., Perkins, K. W., Blackburn, C. R. B., Sanford, R., and Cantrill, S. (1964b). Clin. Res., 12, 451 (abstract).

Wöhler, F. (1963). Acta haemat. (Basel), 30, 65.

Zeltmacher, K., and Bevans, M. (1945). Arch. intern. Med., 75, 395. 